\title{
A Critical Period for Activity-Dependent Synaptic Development during Olfactory Bulb Adult Neurogenesis
}

\author{
Wolfgang Kelsch, ${ }^{1,2}$ Chia-Wei Lin, ${ }^{1,2}$ Colleen P. Mosley, ${ }^{2,3}$ and Carlos Lois ${ }^{1,2,3}$ \\ ${ }^{1}$ Picower Institute of Learning and Memory, ${ }^{2}$ Brain and Cognitive Science Department, and ${ }^{3}$ Department of Biology, Massachusetts Institute of Technology, \\ Cambridge, Massachusetts 02139
}

\begin{abstract}
New neurons integrate in large numbers into the mature olfactory bulb circuit throughout life. The factors controlling the synaptic development of adult-born neurons and their connectivity remain essentially unknown. We examined the role of activity-dependent mechanisms in the synaptic development of adult-born neurons by genetic labeling of synapses while manipulating sensory input or cell-intrinsic excitability. Sensory deprivation induced marked changes in the density of input and output synapses during the period when new neurons develop most of their synapses. In contrast, when sensory deprivation started after synaptic formation was complete, input synapses increased in one domain without detectable changes in the other dendritic domains. We then investigated the effects of genetically raising the intrinsic excitability of new neurons on their synaptic development by delivering a voltage-gated sodium channel that triggers long depolarizations. Surprisingly, genetically increasing excitability did not affect synaptic development but rescued the changes in glutamatergic input synapses caused by sensory deprivation. These experiments show that, during adult neurogenesis in the olfactory bulb, synaptic plasticity is primarily restricted to an early period during the maturation of new neurons when they are still forming synapses. The addition of cells endowed with such an initial short-lived flexibility and long-term stability may enable the processing of information by the olfactory bulb to be both versatile and reliable in the face of changing behavioral demands.
\end{abstract}

\section{Introduction}

The main olfactory bulb (OB) in the mammalian brain receives new neurons throughout life (Altman, 1962; Lois and AlvarezBuylla, 1993; Luskin, 1993). All new neurons added to the adult olfactory bulb are interneurons (Lledo et al., 2008); 95\% of these are granule cell neurons (GCs), and the rest are periglomerular neurons. Considerable progress has been made recently in identifying the factors that regulate the specification of the different subtypes of adult-born interneurons in the olfactory bulb (Hack et al., 2005; Kelsch et al., 2007; Merkle et al., 2007; Batista-Brito et al., 2008). In contrast, relatively little is known about the mechanisms that regulate the synaptic development of adult-born neurons and their connectivity within the mature circuit.

Neuronal activity is thought to be an important factor shaping the wiring of new neurons. In neonatal animals, sensory deprivation reduces the survival of new GCs (Brunjes, 1994; Saghatelyan et al., 2005) and triggers a decrease in spine density and excitatory inputs (Saghatelyan et al., 2005), whereas preexisting, mature GCs maintain their spine density (Frazier-Cierpial and Brunjes, 1989; Saghatelyan et al., 2005). Adult-born GCs also have a critical period during their differentiation such that sensory deprivation reduces the survival of new GCs mainly in the third and fourth week after new neurons are

Received May 22, 2009; revised July 9, 2009; accepted July 27, 2009.

This work was supported by the David and Lucille Packard Foundation and by an R01 grant from National Institute on Deafness and Other Communication Disorders (to C.L.). We thank S. Magavi and D. Friedman for their help and C. Goengrich for critically reading the final version of this manuscript.

Correspondence should be addressed to Dr. Carlos Lois, Department of Biology, Massachusetts Institute of Technology, 43 Vassar Street, 46-5263, Cambridge, MA 02139. E-mail: loisc@mit.edu.

D01:10.1523/JNEUROSCI.2406-09.2009

Copyright $\odot 2009$ Society for Neuroscience $\quad$ 0270-6474/09/2911852-07\$15.00/0 born in the subventricular zone (SVZ) (Petreanu and AlvarezBuylla, 2002; Yamaguchi and Mori, 2005). Before this critical period, the survival and dendritic growth of new GCs is independent of sensory input (Petreanu and Alvarez-Buylla, 2002). Interestingly, the beginning of the critical period for survival $[\sim 14 \mathrm{~d}$ after the birth of a new neuron (Yamaguchi and Mori, 2005)] coincides with the time when glutamatergic input synapses start to develop in adultborn GCs (Kelsch et al., 2008).

Here we investigate whether, in addition to the critical period for survival, there exists a critical period during which the synapses of new neurons are plastic. Toward this goal, we used a genetic labeling technique that allowed us to examine how synaptic development is affected by manipulating sensory input and intrinsic excitability of adult-born neurons. To reduce sensory input, we performed unilateral naris occlusion and observed that adult-born neurons that developed in a sensory-deprived bulb experienced synaptic losses in their distal and basal dendritic domains and gain of input sites in the proximal domain. In contrast, when sensory deprivation started after their synaptic development was complete, adult-born GCs only gained synapses in the proximal domain, with no changes in the other domains. Next we examined whether synapse development was affected by manipulating the intrinsic excitability of new GCs. To increase cell-intrinsic neuronal excitability, we genetically delivered $\mathrm{NaChBac}$, a bacterial voltagegated sodium channel (Ren et al., 2001; Luan et al., 2006; Nitabach et al., 2006), into the progenitor cells in the SVZ that give rise to new GCs. Interestingly, this manipulation did not induce detectable synaptic changes in new neurons generated in adult animals under normal conditions but rescued the changes in glutamatergic input synapses induced by sensory deprivation. 
A

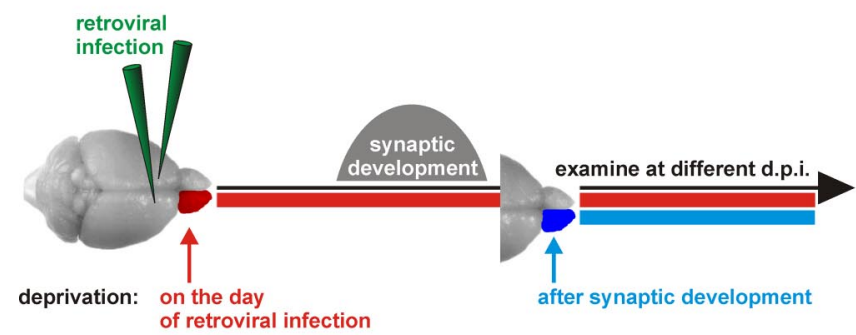

B

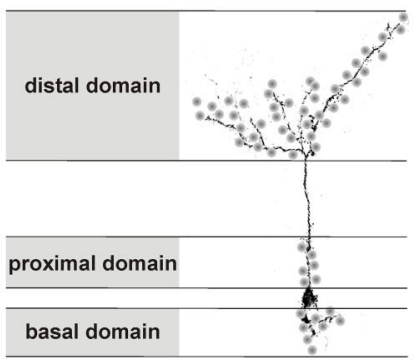

C

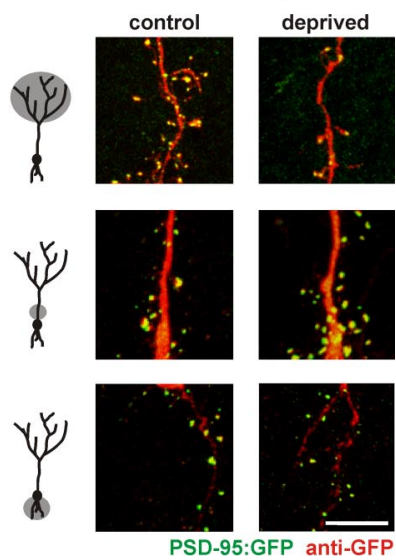

Figure 1. Sensory deprivation changes glutamatergic input synapse development in adult-born GCS. $A$, Progenitor cells were infected with retroviruses in the SVZ in combination with unilateral naris occlusion either at the same day or after synaptic development was complete (starting at $42 \mathrm{dpi}$ ). Genetically labeled $\mathrm{GCS}$ were examined at different days after infection. $\boldsymbol{B}$, $\mathrm{GCs}$ have three main dendritic domains: a basal and a proximal domain that receive glutamatergic input synapses and a distal dendritic domain that contains recurrent input and output synapses. C, At 28 dpi, PSDG ${ }^{+}$ clusters were examined in adult-born GCs in sensory-deprived (starting on the day of retroviral labeling) or contralateral control olfactory bulbs. To attribute PSDG ${ }^{+}$clusters (green) to a particular GC, dendritic morphology was visualized by red dye-labeled GFP immunofluorescence against the diffuse PSD-95:GFP present in the cytosol that was otherwise undetectable. The three main dendritic domains were analyzed separately (from top): distal, proximal, or basal domain. Scale bar, $10 \mu \mathrm{m}$.

These observations indicate that activity-dependent increases or decreases in synaptic densities are primarily restricted to an early period during the maturation of new neurons when they are still forming synapses.

\section{Materials and Methods}

Generation of retroviral vectors. Recombinant retroviral vectors under the control of the Rous sarcoma virus (RSV) promoter for PSDG (PSD-95: GFP) and SypG (synaptophysin:GFP) (Mpsdg and Msypg) were prepared and stored as described previously (Kelsch et al., 2008). All constructs described here were generated by inserting the following cDNAs or cassettes downstream from the RSV promoter in the Molar retroviral vector: Mmcherry, mCherry cDNA; Mnachbacg, a fusion protein between the N terminus of $\mathrm{NaChBac}$ and the $\mathrm{C}$ terminus of green fluorescent protein (GFP); Mpsdg:2A:nachbac, a bicistronic cassette encoding a fusion between postsynaptic density 95 (PSD-95) and GFP linked by a picornavirus 2A sequence to the NaChBac cDNA; Msypg:2A:nachbac, a bicistronic cassette encoding a fusion between synaptophysin and GFP linked by a picornavirus 2A sequence to the NaChBac cDNA; Mpsdg:2A:e191k, a bicistronic cassette encoding a fusion between PSD-95 and GFP linked by a picornavirus 2A sequence to the nonconducting e191k mutant of NaChBac.

Retroviral labeling in vivo. All experiments were performed with 2-month-old Sprague Dawley rats. All animal procedures were approved by the local Animal Welfare Committee and National Institutes of Health guidelines. Retroviral injections were performed as described recently (Kelsch et al., 2008). The stereotaxic coordinates are in millimeters in reference to bregma: anterior, +1.2 ; lateral, \pm 1.6 ; ventral, -3.1 . Animals were kept in a $12 \mathrm{~h}$ daylight cycle and under the same housing conditions.

Olfactory deprivation. For sensory deprivation experiments, unilateral surgical naris occlusion was performed on the day of intracerebral injection of viruses $\mathrm{M} p s d g, \mathrm{Msypg}$, and Mpsdg:2A:nachbac into the subventricular zone. On the day of surgery, adult rats obtained a single intraperitoneal injection of bromodeoxyuridine (BrdU) $(100 \mathrm{mg} / \mathrm{kg}$ bodyweight) to monitor the success of sensory deprivation by an external criterion (Yamaguchi and Mori, 2005). Only animals were included in the analysis of early deprivation that displayed a significant decrease in BrdU-positive $\left(\mathrm{BrdU}^{+}\right)$cells in the deprived bulb (supplemental Fig. 1, available at www.jneurosci.org as supplemental material). As expected (Yamaguchi and Mori, 2005), after late deprivation, no decrease in $\mathrm{BrdU}^{+}$cells in the deprived bulb (BrdU injection at the day of retroviral infection) was observed $(0.98 \pm 0.07)$, but the deprived site displayed a strong reduction in c-Fos expression in the granule cell layer and reduction of tyrosine hydroxylase expression in the glomerular layer (supplemental Fig. 1, available at www.jneurosci.org as supplemental material). For this selection, process immunofluorescence was performed with antibodies against BrdU (Accurate), c-Fos (Oncogene), and tyrosine hydroxylase (Millipore Bioscience Research Reagents).

Analysis of synaptic markers. Tissue processing and analysis of SypG ${ }^{+}$ and $\mathrm{PSDG}^{+}$clusters was performed as described previously (Kelsch et al., 2008). In brief, 50- $\mu \mathrm{m}$-thick coronal slices were incubated in primary rabbit anti-GFP (1:4000; Millipore Bioscience Research Reagents) and Alexa-555 secondary antibodies (1:750; Invitrogen). Confocal image stacks were acquired using an Olympus Fluoview confocal microscope ( $60 \times$ oil-immersion lens, 1.4 numerical aperture) (pixel size, $0.23 \times 0.23$ $\mu \mathrm{m}, 1024 \times 1024$ pixel) and with $z$-step of $0.25 \mu \mathrm{m}$ (80-150 sections). Maximal intensity projections were used to measure the density of $\mathrm{PSDG}^{+}$or SypG ${ }^{+}$clusters of a dendritic segment with the integrated morphometry analysis of MetaMorph software (Molecular Devices).

Statistical analysis. Each analyzed data point [e.g., sensory deprivation and basal domain, $17 \mathrm{~d}$ postinfection (dpi)] contained normally distributed PSDG ${ }^{+}$cluster densities from 14 cells. GCs with deep and superficial dendritic targeting in the external plexiform layer (Kelsch et al., 2007) showed the same activity-dependent plasticity in their dendritic domains (data not shown); therefore, presented data were pooled. Statistical significance was determined using a Student's $t$ test for pairwise comparisons at the same days postinfection.

Electrophysiological recordings. Whole-cell recordings were performed as described previously (Kelsch et al., 2007, 2008). In brief, $350 \mu \mathrm{m}$ horizontal acute slices were prepared from adult olfactory bulbs and recovered in recording solution (in $\mathrm{mM}$ ): $125 \mathrm{NaCl}, 2.5 \mathrm{KCl}, 1.25$ $\mathrm{NaH}_{2} \mathrm{PO}_{4}, 26 \mathrm{NaHCO}_{3}, 1 \mathrm{MgCl}_{2}, 2 \mathrm{CaCl}_{2}$, and 20 glucose, pH 7.3 (312 $\mathrm{mOsm})$. Fluorescence-guided whole-cell patch-clamp recordings were performed and analyzed with a MultiClamp 700B amplifier and pClamp9 software (Molecular Devices). The pipette solution contained the following (in $\mathrm{mm}$ ): $2 \mathrm{NaCl}, 4 \mathrm{KCl}, 130$ Kgluconate, 10 HEPES, 0.2 EGTA, 4 ATP-Mg, 0.3 GTP-Tris, and 14 phosphocreatine, $\mathrm{pH} 7.3$ with $\mathrm{KOH}$. Access resistance was $<20 \mathrm{M} \Omega$, and junction potential was not corrected. To determine the current-voltage relationship of $\mathrm{NaChBac}-$ expressing GCs, $1 \mu \mathrm{M}$ tetrodoxin was used. Because fluorescence of the fusion protein was too weak to detect constructs containing both $\mathrm{NaChBac}$ and a synaptic marker in acute slices, in vitro retroviral expression in human embryonic kidney cell lines was used to confirm that the current was pre- 
served. A slow inactivating inward current was activated by depolarization as described previously (Ren et al., 2001) for Mpsdg:2A:nachbac and Msypg:2A:nachbac but not for mutant Mpsdg:2A: e191k (data not shown).

\section{Results}

Adult-generated neurons display different synaptic changes in specific dendritic domains in response to sensory deprivation

To determine how neuronal activity affects the synaptic development of adultborn neurons in the rat olfactory bulb, we blocked sensory input to the bulb by performing unilateral naris occlusion (Fig. $1 A)$ and compared the synaptic structure and organization of GCs in the deprived and contralateral control olfactory bulb. We measured the development of glutamatergic input synapses of new adultborn GCs using PSDG, a genetic marker consisting of a fusion protein between PSD-95 and GFP. PSD-95 is a protein localized to the postsynaptic density of glutamatergic input synapses (Sheng, 2001), and PSDG delivered into new neurons with retroviral vectors $(\mathrm{M} p s d g$ ) can be used to genetically label these synapses (Niell et al., 2004; Gray et al., 2006; Kelsch et al., 2008; Livneh et al., 2009). We have shown previously that expression of PSDG at the modest levels yielded by retroviral expression does not alter synaptic properties as measured by electrophysiological methods (Kelsch et al., 2008). To analyze the development of output synapses, we used SypG, a fusion protein between synaptophysin and GFP. Synaptophysin is a protein localized to presynaptic neurotransmitter vesicles (Südhof and Jahn, 1991), and SypG expressed with retroviral vectors (Msypg) can be used to genetically label output synapses ( $\mathrm{Li}$ and Murthy, 2001; Meyer and Smith, 2006; Kelsch et al., 2008). We examined SypG ${ }^{+}$cluster densities at 28 dpi, once GCs had completed their maturation and SypG ${ }^{+}$clusters were confined to spines of the distal dendritic domain (supplemental Fig. 1, available at www.jneurosci.org as supplemental material).

GCs are axonless inhibitory interneurons, and their synapses are distributed in three dendritic domains, know as basal, proximal, and distal dendritic domains (Fig. 1B). GCs receive glutamatergic input synapses through the basal, proximal, and distal domains. The distal dendritic domain of GCs contains both input and output synapses, whereas the basal and proximal dendritic domains do not release neurotransmitter (Mori, 1987).

Blocking sensory input during a period in which new neurons are undergoing synaptic development, between 14 and $28 \mathrm{dpi}$, led to reductions in the density of $\mathrm{PSDG}^{+}$input synapses (Fig. 1C) in the distal and basal dendritic domains (Fig. 2A). Similarly, the density of SypG ${ }^{+}$output synapses in the distal dendritic domain decreased significantly under sensory deprivation (Fig. 3). Interestingly, sen-

$$
\begin{gathered}
\overrightarrow{-\overrightarrow{p s d g}-} \\
\text { control } \\
\text { vs. } \\
\text { deprivation }
\end{gathered}
$$
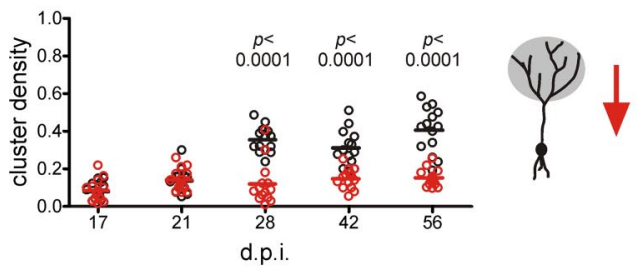
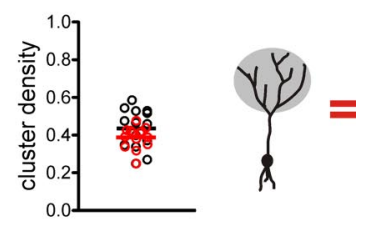

deprivation after synaptic development
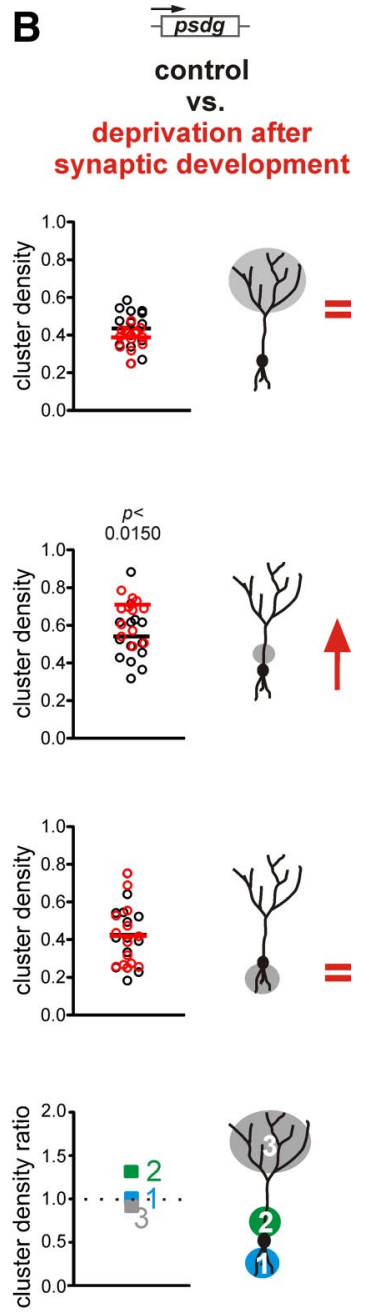

Figure 2. Sensory deprivation changes glutamatergic synapses in specific dendritic domains during a critical period. $A$, Scatter plot and mean density of PSDG ${ }^{+}$clusters (clusters per micrometer) of adult-born GCs in sensory-deprived (starting on the day of retroviral labeling) and contralateral control (red and black circles, respectively) olfactory bulbs at different days after infection. domain (unilateral naris occlusion at $42 \mathrm{dpi}$ and examined at $63 \mathrm{dpi}$ ).

sory deprivation during synaptic development increased the density of $\mathrm{PSDG}^{+}$input synapses in the proximal dendritic domain of new GCs (Fig. 2A). The changes in synaptic densities became statistically significant at the time when new neurons reached maturity (28 dpi) and remained so thereafter until the longest time we monitored the new neurons (56 dpi) (Fig. $2 \mathrm{~A}$ ). For this and the following experiments, data from GCs with different dendritic targeting were pooled as an initial analysis and indicated no significant differences between these subpopulations. These observations indicate that sensory deprivation to the bulb triggered upregulation and downregulation of the synaptic input and outputs of new GCs. In addition, these activity-dependent synaptic changes were not uniform throughout the cells but confined to specific dendritic domains.

We next examined whether changes in synaptic organization also occurred when sensory deprivation was started after synaptic development of new GCs was complete. Toward this end, unilateral naris occlusion was started $42 \mathrm{~d}$ after a cohort of genetically labeled new neurons had been born in the SVZ (Fig. 1A). Adult-born GCs de- 
A

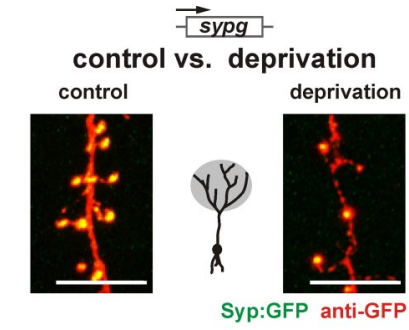

B

control
vs.

deprivation

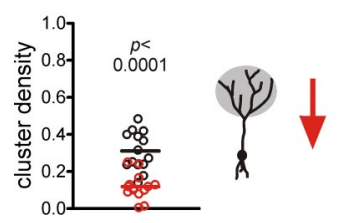

C

contro

vs.

deprivation

after synaptic development

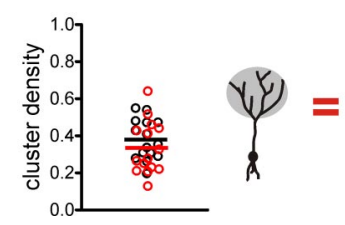

Figure 3. Sensory deprivation during synaptic development reduces output synapse density. A, At 28 dpi, synaptophysin:GFP ${ }^{+}$clusters were examined in the distal domain of adultborn GCs from sensory-deprived (starting on the day of retroviral labeling) and contralateral control olfactory bulbs. Scale bars, $10 \mu \mathrm{m}$. B, Scatter plot and mean density of SypG ${ }^{+}$clusters (clusters per micrometer) of adult-born GCs from sensory-deprived (starting on the day of retroviral labeling) and contralateral control (red and black circles, respectively) olfactory bulbs at $28 \mathrm{dpi}$ ( $t$ test). C, When sensory deprivation started after synaptic development was complete, there were no changes in the mean density of SypG ${ }^{+}$clusters (clusters per micrometer) (unilateral naris occlusion at $42 \mathrm{dpi}$ and examined at $63 \mathrm{dpi}$ ).

prived of sensory input for 3 weeks (between 42 and 63 dpi), starting after completion of synaptic development of a cohort of labeled neurons, only resulted in an increase in synaptic input in the proximal domain (Fig. $2 B$ ), with no detectable changes in synaptic input or output densities in the distal and basal domains (Figs. $2 B, 3$ ). These experiments suggest the existence of a critical period during the synaptic development of new neurons when the organization of synapses throughout the cell can be strongly shaped by sensory experience. In addition, these results indicate that the proximal dendritic domain of new neurons has special properties that enable it to alter its synaptic organization after all other dendritic domains become unresponsive to manipulations of sensory input.

\section{A genetic method to increase cell-intrinsic excitability}

The activity of neurons is primarily regulated by two factors, the synaptic input that they receive and their intrinsic excitability determined by the set of ion channels present in their membranes. To investigate the role of intrinsic excitability in synaptic development, we tested whether increasing cell-intrinsic excitability would change the formation and maintenance of synapses as they integrate into the circuits of the bulb. Toward this goal, we devised a genetic strategy that would selectively render these new neurons more excitable without significantly altering the properties of the rest of the circuit into which they integrate. To increase the intrinsic excitability of single neurons, we used $\mathrm{NaChBac}$, a voltage-gated sodium channel from the hallophilic bacteria Bacillus halodurans. Expression of $\mathrm{NaChBac}$ increases the intrinsic excitability of neurons because, first, its activation threshold is more negative than that of native sodium channels, and second, $\mathrm{NaChBac}$ produces a slowly inactivating depolarizing current that lasts on the order of hundreds of milliseconds (Ren et al., 2001) compared with the $\sim 1 \mathrm{~ms}$ inactivation time of the endogenous mammalian sodium channels responsible for the fast action potentials (Llinas, 1988). To visualize neurons expressing $\mathrm{NaChBac}$, we generated a retroviral vector encoding a fusion protein
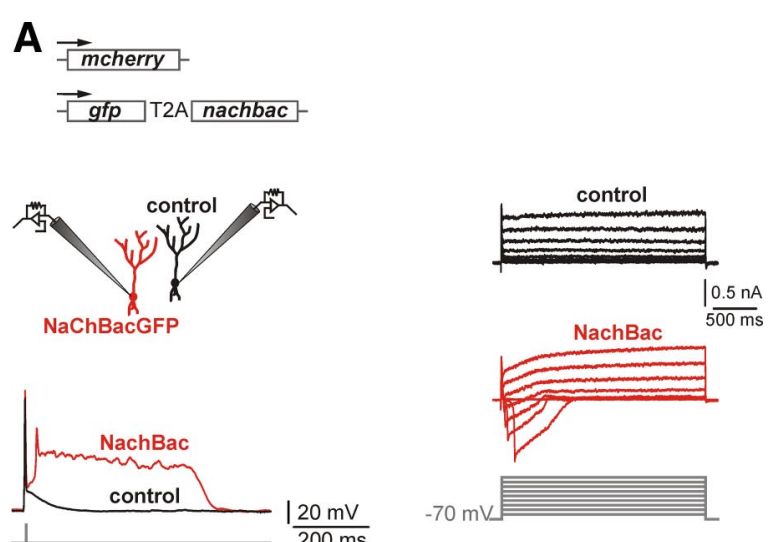

B

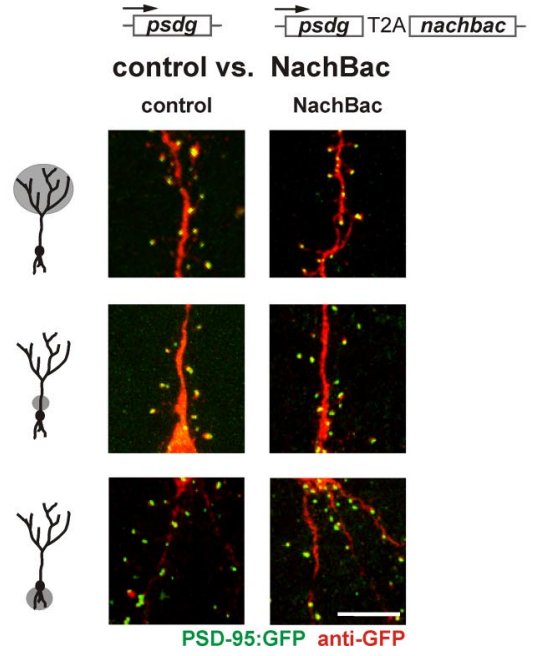

Figure 4. Genetically increased excitability does not change glutamatergic input synapse development. $\boldsymbol{A}$, Whole-cell recordings were obtained from acute slices containing $\mathrm{GCs}$ that either expressed mCherry as control or NaChBac:GFP. Bottom left, At $28 \mathrm{dpi}$, a short current injection ( $4 \mathrm{nA}, 5 \mathrm{~ms}$ ) evoked a sustained depolarization in $\mathrm{NaChBac}$ expressing GCs but not in controls of adult-born GCs. Right, The current-voltage relationship revealed a voltage-dependent inward current that was only observed in $\mathrm{NaChBac}$-expressing $\mathrm{GCs}\left(10 \mathrm{mV}\right.$ steps, $\mathrm{V}_{\mathrm{h}}=-70 \mathrm{mV}, 16$ dpi). $\boldsymbol{B}$, At $28 \mathrm{dpi}$, PSDG ${ }^{+}$clusters were examined in adult-born GCs expressing either the synaptic marker alone or with $\mathrm{NaChBac}$. Scale bar, $10 \mu \mathrm{m}$.

between GFP and NaChBac (Mnachbacg). To study the electrical properties of new neurons expressing $\mathrm{NaChBac}$, we delivered Mnachbacg into GC progenitors in the SVZ. As controls, we coinjected a retroviral vector encoding the red fluorescent protein mCherry (Mmcherry) into the same animals and performed fluorescence-guided whole-cell recordings of either mCherry ${ }^{+}$or $\mathrm{NaChBac}^{+}$cells in acute slices. Unlike control neurons ( $n=6 \mathrm{GCs}$ ), $\mathrm{NaChBac}^{+}$neurons expressed a TTX-resistant slow-inactivating inward current that activated at $-40 \mathrm{mV}$ ( $n=5$ GCs) (Fig. $4 \mathrm{~A}$ ). $\mathrm{NaChBac}$ expression did not alter other passive membrane properties measured at $-70 \mathrm{mV}$ such as membrane time constant, input resistance, and resting membrane potential (data not shown). Whereas a $5 \mathrm{~ms}$ pulse of suprathreshold current injection $(4 \mathrm{nA})$ evoked a single spike in mCherry ${ }^{+}$control GCs ( $n=9$ GCs), the same current injection reliably caused an additional depolarization lasting on average $566 \pm 49 \mathrm{~ms}$ in $\mathrm{NaChBac}^{+} \mathrm{GCs}(n=8 \mathrm{GCs}$ ) (Fig. $4 A$ ). This suprathreshold current injection ( $4 \mathrm{nA}, 5 \mathrm{~ms}$ ) elicited long depolarizations in $\mathrm{NaChBac}^{+}$GCs as early as $14-18$ dpi $(n=8$ GCs), which were never observed in control neurons at any of the time points studied. At $28 \mathrm{dpi}$, the activation threshold for spiking in $\mathrm{NaChBac}^{+}$GCs was $-41 \pm 2.0 \mathrm{mV}(n=9 \mathrm{GCs})$, whereas for mCherry $^{+}$, control GCs was $-30 \pm 1.9 \mathrm{mV}(n=8 \mathrm{GCs})$ measured 
at 26-28 dpi in adult rats. $\mathrm{NaChBac}$ expression did not affect the migration of new neurons into the olfactory bulb and increased their survival (our unpublished observations).

\section{Genetically increasing the intrinsic} excitability of adult-born neurons does not affect synapse formation Having established a method to genetically increase the intrinsic excitability of newly generated neurons, we examined how this manipulation affected the formation of synapses using a bicistronic construct that expressed both PSDG, the fusion between GFP and PSD-95, and $\mathrm{NaChBac}$ (Mpsdg: 2A:nachbac) (Fig. 4B). We measured the density of PSDG ${ }^{+}$synapses in new GCs infected with Mpsdg:2A:nachbac and compared them with neurons expressing PSDG either alone (Fig. $4 B$ ) or with a nonconducting $E 191 \mathrm{~K} \mathrm{NaChBac}$ mutant (Mpsdg:2A: e191k) (supplemental Fig. 1, available at www.jneurosci.org as supplemental material). GCs expressing both PSDG and the nonconducting $E 191 \mathrm{~K} \mathrm{NaChBac}$ mutant (Mpsdg:2A:e191k) had the same PSDG ${ }^{+}$ synapse density as control neurons expressing only PSDG in adult-born GCs (supplemental Fig. 1, available at www. jneurosci.org as supplemental material), indicating that expression of the nonconducting channel did not change PSDG ${ }^{+}$ synapse density by itself. To examine whether increased membrane excitability affected output synapses, we generated a bicistronic construct that expressed both synaptophysin:GFP (Sypg), the fusion protein between synaptophysin and GFP, and NaChBac (Msypg:2A:nachbac) (see Fig. 6).

Unexpectedly, increasing the intrinsic excitability by $\mathrm{NaChBac}$ expression in adult-born GCs did not change the density of $\mathrm{PSDG}^{+}$synapses in any of their dendritic domains (Fig. 5A), although $\mathrm{NaChBac}$ remained active during synaptic development of adult-born GCs (Fig. $4 A)$. Similarly, the density of SypG ${ }^{+}$output synapses in adult-generated GCs was not changed by $\mathrm{NaChBac}$ expression (Msypg:2A:nachbac vs Msypg) (Fig. 6). These observations indicate that the formation of synapses is not affected by perturbations in the cell-intrinsic excitability of new neurons.

\section{Increased intrinsic excitability}

blocks the synaptic changes evoked by sensory deprivation

The observation that strong synaptic changes were triggered by sensory deprivation, but not by raising the intrinsic excitability of new neurons could be at-

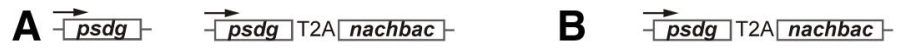

control vs. NachBac

NachBac vs. $\mathrm{NaChBac}+$ deprivation
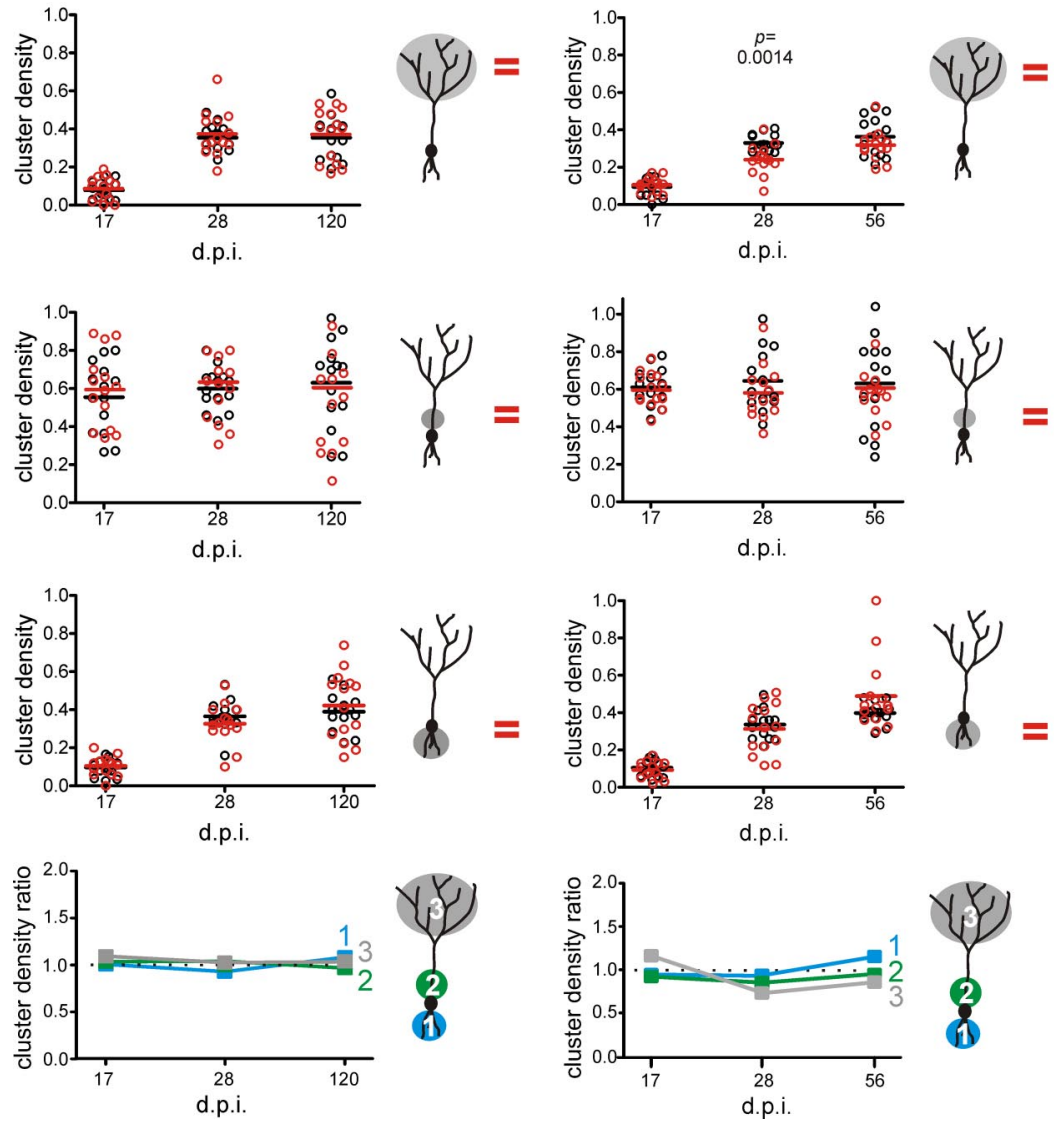

Figure 5. Genetically increased excitability does not change glutamatergic input synapse development and blocks the synaptic changes induced by sensory deprivation. $A$, Scatter plot and mean density of PSDG ${ }^{+}$clusters (clusters per micrometer) in a dendritic domain of new control or $\mathrm{NaChBac}$-expressing $\mathrm{GCs}$ (black and red circles, respectively) born in the adult and examined at different dpi. Statistical significance is only indicated if $p<0.05$ ( $t$ test). The bottom graph shows the ratio of the mean cluster density of $\mathrm{NaChBac}$-expressing over control GCs in a specific dendritic domain at a given day after infection. $\boldsymbol{B}$, Same as $\boldsymbol{A}$, but instead, adult-born GCs coexpressed PSD-95:GFP and NaChBac in both the sensorydeprived (starting on the day of retroviral labeling) and contralateral control olfactory bulb. Statistical significance is only indicated if $p<0.05$ ( $t$ test).
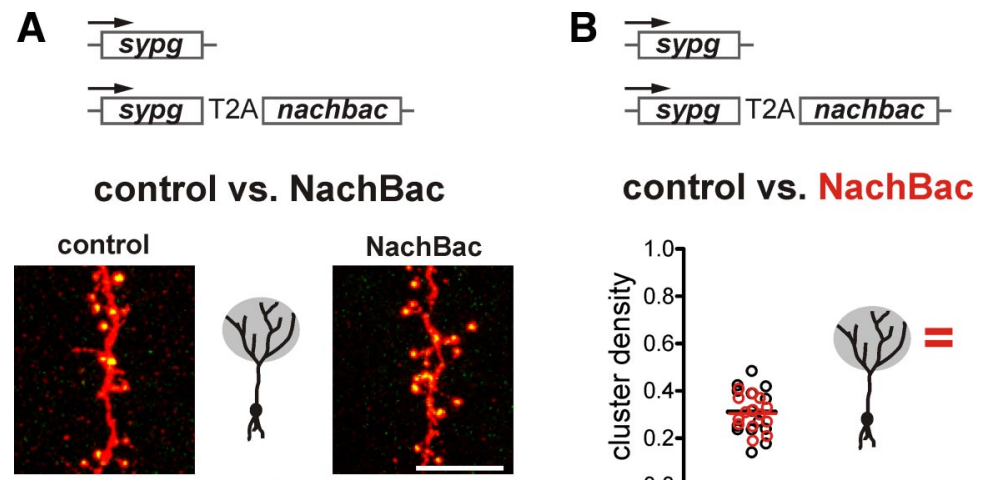

Figure 6. Genetically increased excitability does not change output synapse development. $\boldsymbol{A}$, At $28 \mathrm{dpi}$, SypG ${ }^{+}$clusters were examined in the distal domain of adult-born GCs expressing either the synaptic marker alone or with $\mathrm{NaChBac}$. Scale bar, $10 \mu \mathrm{m}$. B, Scatter plot and mean density of SypG ${ }^{+}$clusters (clusters per micrometer) of new control or NaChBacexpressing GCs (black and red circles, respectively) born in the adult at $28 \mathrm{dpi}$. No significant differences were detected ( $t$ test). 


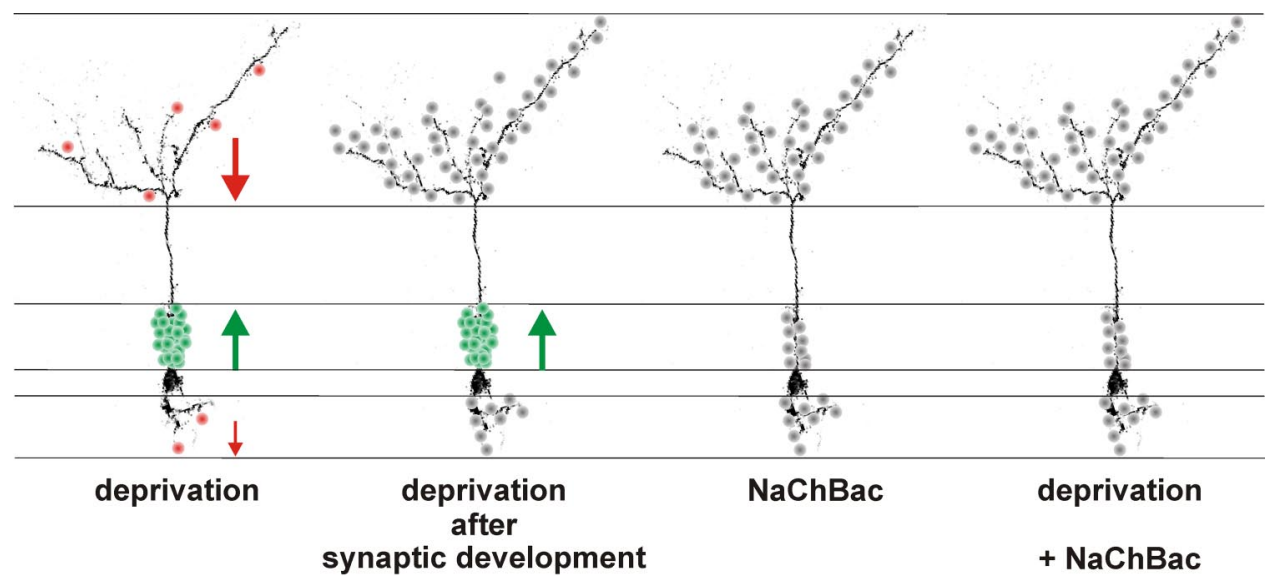

Figure 7. Sensory deprivation and intrinsic excitability differently control synaptic development in the dendritic domains of adult-born neurons. Reduced sensory input during synaptic development changed synaptic densities in all dendritic domains. When sensory deprivation started after the completion of synaptic development, the only detectable changes were increases in the density of glutamatergic input synapses in the proximal domain. Genetically increasing the excitability of new neurons did not affect the synaptic development of cells that matured in a normal sensory stimulation but rescued the synaptic changes triggered by sensory deprivation.

tributable to the ability of these new neurons to implement mechanisms of plasticity when their synaptic inputs are modified (by sensory deprivation) but not when their intrinsic membrane excitability is perturbed (by $\mathrm{NaChBac}$ expression). Alternatively, synaptic changes attributable to increased excitability of adultgenerated neurons may only come into play if their synaptic inputs are perturbed. To test these possibilities, we analyzed the distribution of synapses in new $\mathrm{NaChBac}^{+}$adult-born GCs that had also been deprived of sensory input. As indicated above, sensory deprivation caused a decrease in the number of glutamatergic input synapses in the distal and basal dendritic domains of adult-born neurons and an increase in the proximal domain (Fig. 2). Surprisingly, NaChBac blocked the changes in $\mathrm{PSDG}^{+}$synapse densities of adult-born neurons triggered by sensory deprivation. In the proximal domain, $\mathrm{NaChBac}$ expression completely eliminated the synaptic increases induced by sensory deprivation at all times studied (Fig. 5B). In the distal domain, NaChBac expression blocked the decrease in synaptic density induced by naris occlusion partially by $28 \mathrm{dpi}$ and completely by $56 \mathrm{dpi}$ (Fig. $5 B$ ). These observations indicate that increasing the intrinsic excitability of new neurons in the adult brain can trigger synaptic changes in glutamatergic input synapses but only after their activity is modified by sensory deprivation.

\section{Discussion}

The connectivity of neurons within brain circuits is primarily regulated at two levels: first, by determining the identity of the neurons that will become synaptic partners, and second, by controlling the number of synapses formed between these partners. During adult neurogenesis in the $\mathrm{OB}$, the identity of the synaptic partners for new GCs (mitral or tufted cells) appears to be primarily determined by genetic factors, such that separate precursors exist in the SVZ that give rise to new GCs apparently committed to make synapses with either mitral or tufted cells (Kelsch et al., 2007). In contrast, the density of synaptic connections can be strongly regulated by neuronal activity (Brunjes, 1994; Saghatelyan et al., 2005). Such a dual control of neuronal connectivity during adult neurogenesis may simultaneously provide stereotypy of the circuit and flexibility to form novel connections for processing information in response to sensory-driven behavioral demands.
In this study, we analyzed how neuronal activity sculpts the synaptic development and connectivity of new GCs added to an adult brain circuit. We observed that new neurons added to the adult olfactory bulb under sensory deprivation experience dramatic changes in their synaptic development. In particular, we focused in this study on how and when neuronal activity can change the density and distribution of synaptic input and output sites of adult-born neurons. These changes are non-uniform in that either increases or decreases in synapse density occurred in different dendritic domains (Fig. 7). Sensory deprivation caused decreases in synapses in the distal and basal domain and increases in the proximal domain. Thus, each dendritic domain appears to act as an independent unit of synaptic plasticity.

Interestingly, sensory input-dependent changes in synaptic wiring are primarily restricted to the period when the new adultborn neurons develop their synapses. The synapses in the distal and basal domain are modifiable by sensory activity only for a limited time during the maturation of new GCs. After GCs have completed their maturation, the only synapses that appear to retain their modifiability in response to sensory deprivation are located in the proximal dendritic domain. In line with the limited period of activity-dependent synaptic changes that we observed, it has been shown recently that the ability to induce long-term potentiation was also lost after the first month in the life of new adult-born GCs (Nissant et al., 2009).

Given the strong synaptic changes induced by sensory deprivation on new GCs, it is surprising that genetically triggering long depolarizations $(\sim 600 \mathrm{~ms})$ in these new neurons throughout their maturation did not interfere with the formation and maintenance of synapses. Despite the strong electrical perturbation induced by $\mathrm{NaChBac}$ expression, we could not detect any morphological changes in the synapse in any of the dendritic domains of new GCs. Similarly, the frequency and amplitudes of spontaneous EPSC inputs of adult-born GCs expressing $\mathrm{NaChBac}$ were not altered compared with matched controls (our unpublished observations). These results were surprising because many neurons have homeostatic mechanisms that regulate their synaptic input so that, when their excitability is raised, they reduce the number or strength of their excitatory synapses to prevent runaway activity. Interestingly, although GCs in the OB do not exhibit this compensatory behavior, we have recently observed that 
new neurons in the dentate gyrus with $\mathrm{NaChBac}$-enhanced excitability experience a strong reduction in their excitatory synapses (our unpublished results). The observation that increased excitability does not affect the density or distribution of excitatory synapses in GCs in the OB could be explained by other compensatory mechanisms, such as increased synaptic inhibition or changes in ion channels that could balance the increased intrinsic excitability. However, we did not observe any changes in intrinsic membrane or synaptic properties, suggesting either the existence of subtle compensatory mechanisms that escaped our detection methods or, alternatively, that these neurons do not compensate for this form of increased cell-intrinsic excitability.

Adult-born neurons, however, were not completely unresponsive to manipulations of their intrinsic excitability, because $\mathrm{NaChBac}$-enhanced excitability counteracted the changes in glutamatergic input synapses caused by sensory deprivation. These observations also suggest the possibility that there may exist a program regulating the formation and maintenance of synapses in new GCs that requires a minimum threshold of neuronal activity contributed by the combined action of synaptic drive and cell-intrinsic excitability. If neuronal activity falls below that putative threshold attributable to a reduction in synaptic inputs (as a result of sensory deprivation), new neurons implement changes in the organization of their synapses. If new GCs with reduced synaptic input are simultaneously rendered hyperexcitable (as a result of $\mathrm{NaChBac}$ expression), the cells may still be over the threshold so that no synaptic changes occur.

The ability of new neurons to adapt their connectivity to the state of the adult circuit (e.g., sensory deprivation vs normal environment) may shed light on the contribution of continuous neurogenesis to learning and memory (Nottebohm, 2002; Lledo and Saghatelyan, 2005; Bischofberger, 2007; Aimone et al., 2009). Several studies have demonstrated that the plasticity of adultborn neurons is maximal during their initial development and that it is progressively lost as the cells mature (Schmidt-Hieber et al., 2004; Ge et al., 2007; Nissant et al., 2009). Our results extend these observations by demonstrating that the synapses of new neurons have a high degree of structural plasticity during a time window when they are initially added into the circuit. Once they mature, activity-dependent plasticity in new neurons becomes more limited. This window of plasticity may allow the bulb to capture new neurons into stable patterns of connectivity while the circuit is in a certain state and thus generate stable information processing modules, whereas the next wave of new neurons may capture subsequent "circuit states." The addition of cells endowed with such an initial short-lived flexibility and long-term stability may enable the processing of information by the olfactory bulb to be both versatile and reliable in the face of changing behavioral demands.

\section{References}

Aimone JB, Wiles J, Gage FH (2009) Computational influence of adult neurogenesis on memory encoding. Neuron 61:187-202.

Altman J (1962) Are new neurons formed in the brains of adult mammals? Science 135:1127-1128.

Batista-Brito R, Close J, Machold R, Fishell G (2008) The distinct temporal origins of olfactory bulb interneuron subtypes. J Neurosci 28:3966-3975.

Bischofberger J (2007) Young and excitable: new neurons in memory networks. Nat Neurosci 10:273-275.

Brunjes PC (1994) Unilateral naris closure and olfactory system development. Brain Res Brain Res Rev 19:146-160.

Frazier-Cierpial LL, Brunjes PC (1989) Early postnatal differentiation of granule cell dendrites in the olfactory bulbs of normal and unilaterally odor-deprived rats. Brain Res Dev Brain Res 47:129-136.

Ge S, Yang CH, Hsu KS, Ming GL, Song H (2007) A critical period for enhanced synaptic plasticity in newly generated neurons of the adult brain. Neuron 54:559-566.

Gray NW, Weimer RM, Bureau I, Svoboda K (2006) Rapid redistribution of synaptic PSD-95 in the neocortex in vivo. PLoS Biol 4:e370.

Hack MA, Saghatelyan A, de Chevigny A, Pfeifer A, Ashery-Padan R, Lledo PM, Götz M (2005) Neuronal fate determinants of adult olfactory bulb neurogenesis. Nat Neurosci 8:865-872.

Kelsch W, Mosley CP, Lin CW, Lois C (2007) Distinct mammalian precursors are committed to generate neurons with defined dendritic projection patterns. PLoS Biol 5:e300.

Kelsch W, Lin CW, Lois C (2008) Sequential development of synapses in dendritic domains during adult neurogenesis. Proc Natl Acad Sci U S A 105:16803-16808.

Li Z, Murthy VN (2001) Visualizing postendocytic traffic of synaptic vesicles at hippocampal synapses. Neuron 31:593-605.

Livneh Y, Feinstein N, Klein M, Mizrahi A (2009) Sensory input enhances synaptogenesis of adult-born neurons. J Neurosci 29:86-97.

Lledo PM, Saghatelyan A (2005) Integrating new neurons into the adult olfactory bulb: joining the network, life-death decisions, and the effects of sensory experience. Trends Neurosci 28:248-254.

Lledo PM, Merkle FT, Alvarez-Buylla A (2008) Origin and function of olfactory bulb interneuron diversity. Trends Neurosci 31:392-400.

Llinás RR (1988) The intrinsic electrophysiological properties of mammalian neuons: insights into central nervous system. Science 242:1654-1664.

Lois C, Alvarez-Buylla A (1993) Proliferating subventricular zone cells in the adult mammalian forebrain can differentiate into neurons and glia. Proc Natl Acad Sci U S A 90:2074-2077.

Luan H, Lemon WC, Peabody NC, Pohl JB, Zelensky PK, Wang D, Nitabach MN, Holmes TC, White BH (2006) Functional dissection of a neuronal network required for cuticle tanning and wing expansion in Drosophila. J Neurosci 26:573-584.

Luskin MB (1993) Restricted proliferation and migration of postnatally generated neurons derived from the forebrain subventricular zone. Neuron 11:173-189.

Merkle FT, Mirzadeh Z, Alvarez-Buylla A (2007) Mosaic organization of neural stem cells in the adult brain. Science 317:381-384.

Meyer MP, Smith SJ (2006) Evidence from in vivo imaging that synaptogenesis guides the growth and branching of axonal arbors by two distinct mechanisms. J Neurosci 26:3604-3614.

Mori K (1987) Membrane and synaptic properties of identified neurons in the olfactory bulb. Prog Neurobiol 29:275-320.

Niell CM, Meyer MP, Smith SJ (2004) In vivo imaging of synapse formation on a growing dendritic arbor. Nat Neurosci 7:254-260.

Nissant A, Bardy C, Katagiri H, Murray K, Lledo PM (2009) Adult neurogenesis promotes synaptic plasticity in the olfactory bulb. Nat Neurosci 12:728-730.

Nitabach MN, Wu Y, Sheeba V, Lemon WC, Strumbos J, Zelensky PK, White $\mathrm{BH}$, Holmes TC (2006) Electrical hyperexcitation of lateral ventral pacemaker neurons desynchronizes downstream circadian oscillators in the fly circadian circuit and induces multiple behavioral periods. J Neurosci 26:479-489.

Nottebohm F (2002) Why are some neurons replaced in adult brain? J Neurosci 22:624-628.

Petreanu L, Alvarez-Buylla A (2002) Maturation and death of adultborn olfactory bulb granule neurons: role of olfaction. J Neurosci 22:6106-6113.

Ren D, Navarro B, Xu H, Yue L, Shi Q, Clapham DE (2001) A prokaryotic voltage-gated sodium channel. Science 294:2372-2375.

Saghatelyan A, Roux P, Migliore M, Rochefort C, Desmaisons D, Charneau P, Shepherd GM, Lledo PM (2005) Activity-dependent adjustments of the inhibitory network in the olfactory bulb following early postnatal deprivation. Neuron 46:103-116.

Schmidt-Hieber C, Jonas P, Bischofberger J (2004) Enhanced synaptic plasticity in newly generated granule cells of the adult hippocampus. Nature 429:184-187.

Sheng M (2001) Molecular organization of the postsynaptic specialization. Proc Natl Acad Sci U S A 98:7058-7061.

Südhof TC, Jahn R (1991) Proteins of synaptic vesicles involved in exocytosis and membrane recycling. Neuron 6:665-677.

Yamaguchi M, Mori K (2005) Critical period for sensory experiencedependent survival of newly generated granule cells in the adult mouse olfactory bulb. Proc Natl Acad Sci U S A 102:9697-9702. 